\title{
Hydronumerische Modellierung mittels frei verfügbarer Software: ein kurzer Überblick
}

\author{
Sebastian Gegenleithner · Clemens Dorfmann · Josef Schneider (iD
}

Online publiziert: 28. Mai 2020

(c) Der/die Autor(en) 2020

\begin{abstract}
Zusammenfassung In diesem Beitrag wird auf die hydrodynamische Modellierung mit frei verfügbaren Softwarepaketen eingegangen. Besonderes

\section{Numerical hydrodynamic modelling by means of freely available software: a short overview} Augenmerk liegt hierbei auf den Rollen von Personen in der Entwicklung und Anwendung sowie auf deren Stellenwert im Laufe der Zeit. Charakteristiken von frei verfügbarer und kommerzieller Software werden vorgestellt und die jeweiligen Vor- und Nachteile diskutiert. Mittels vier Anwendungsbeispielen werden die Möglichkeiten der Adaptierung von frei verfügbarer Software für neue Entwicklungen bzw. für die Lösung von projektspezifischen Aufgabenstellungen vorgestellt: (i) Berücksichtigung von hydraulischen Verlusten in einem 2D tiefengemittelten Modell, (ii) Implementierung von internen Randbedingungen, (iii) Serielle Kopplung von Modellen, (iv) Auswertung von Zeitreihen mittels Python. Die vorgestellten Überlegungen werden zum Schluss diskutiert und zusammengefasst.

Schlüsselwörter Numerische Modellierung · Historie der Entwicklung von

Modellierungssystemen · Vergleich zwischen freier und kommerzieller Software

DI S. Gegenleithner, BSc $(\bowtie)$ Assoc. Prof. DI Dr. J. Schneider Institut für Wasserbau und Wasserwirtschaft, Technische Universität Graz, Stremayrgasse 10/II, 8010 Graz, Österreich

sebastian.gegenleithner@tugraz.at

DI Dr. C. Dorfmann

flow engineering,

Brockmanngasse 108, $8010 \mathrm{Graz}$, Österreich
Abstract This article deals with hydrodynamic modelling by means of freely available software packages. Particular attention is paid to the role of developers and users as well as their involvement over time. The main characteristics of commercial and freely available software are presented and respectively, their advantages and disadvantages are discussed. Four applications of freely available software packages are presented, namely: (i) consideration of hydraulic losses in a 2D depth-averaged model, (ii) implementation of internal boundary conditions, (iii) serial coupling of modelling software and (iv) evaluation of time series using Python. Finally, the main aspects and considerations are discussed and summarised.

Keywords Numerical modelling · History of modelling systems .

Comparison between freely available and commercial software

\section{Einleitung}

Die Berechnung hydrodynamischer Prozesse mit mathematischen Modellen zählt heutzutage zu einer der Hauptaufgaben sowohl in der Grundlagenforschung als auch in der wasserbaulichen Praxis. Die Auswahl der geeigneten Modellierungssoftware für die jeweilige Fragestellung hängt dabei von einer Vielzahl von Faktoren ab. Typische Überlegungen bei der Auswahl sind mitunter: (i) Welche physikalischen Prozesse sollen abgebildet werden? So werden z. B. für kleinskalige Detailuntersuchungen der Strömung auf den Navier-Stokes-Gleichungen basierende dreidimensionale (3D) Modelle eingesetzt, wohingegen für großskalige Anwendungen zweidimensionale (2D) tiefengemittelte oder eindimensiona- le (1D) Modelle angewandt werden; (ii) Finanzielle Betrachtungen, oder auch; (iii) Soll kommerzielle oder frei verfügbare Software für die gegebene Aufgabenstellung eingesetzt werden?

Kommerzielle (zahlungspflichtige) und frei verfügbare Software (Freeware, Open-Source-Software) lässt sich wie folgt unterscheiden:

Kommerzielle Software: Software, die von einem Unternehmen mit dem Ziel entwickelt wird, aus der Benutzung Profit zu machen. Häufig wird Anwendenden ein hoher Serviceumfang (z. B. Hilfestellungen bei der Bedienung etc.) geboten.

Freeware: Als Freeware wird Software bezeichnet, die unentgeltlich benutzt werden kann. Der Quellcode kann nicht eingesehen oder modifiziert werden.

Open-Source-Software (Freie Software): Laut Definition der Open Source Initiative muss eine unter dem Open-Source-Lizenzmodell angebotene Software zehn strenge Kriterien erfüllen (Open Source Initiative 2007). Aus einem Artikel von Richard Stallman (DiBona und Ockman 1999) können hingegen folgende drei Hauptkriterien oder Freiheiten für die Charakterisierung von Freier Software kondensiert werden: (i) Das Programm kann für jegliche Anwendung eingesetzt werden; (ii) Der Quellcode der Software kann nach Belieben verändert werden; (iii) Die Software darf sowohl unverändert als auch in einer veränderten Form beliebig weitergegeben werden.

Im Folgenden wird ein kurzer Überblick über die historische Entwicklung von Modellierungssoftware gegeben. Anschließend werden Charakteristiken von frei verfügbarer und kommerzieller Software beschrieben und einander gegenübergestellt. Anhand von vier Beispielen werden schlussendlich die Möglichkeiten der Adaptierung frei verfügbarer Software für neue Entwicklungen bzw. für die Lösung von projektspezifischen Aufgabenstellungen vorgestellt. 


\section{Verschiedene Generationen von Modellierungssoftware}

Mitte der 1940er-Jahre erlebte die Entwicklung elektronischer Rechenmaschinen, später Computer genannt, einen immensen Aufschwung (Copeland 2006). Schon damals wurden erste Mehrzweckrechner entwickelt, die in der Lage waren, einfache Programme auszuführen. Diese Programme wurden in Form gestanzter Lochkarten an die Maschine übergeben. Klassischen Programmcode, wie man ihn heutzutage kennt, gab es bis zur Entwicklung der ersten „High Level“-Programmiersprache Fortran im Jahr 1954 jedoch kaum. Seit diesen Pionierarbeiten ist ein immenser Fortschritt in der Entwicklung von Software und Hardware erkennbar. So besagt das Moore'sche Gesetz (Moore 1965), dass sich die Komplexität von Schaltkreisen und infolgedessen auch die Rechenleistung in Zeitintervallen von ca. 18 Monaten verdoppelt.

Mit dem exponentiellen Anstieg der Computerleistung und der Entwicklung von „High Level“-Programmiersprachen hat der Computer auch mehr und mehr Einzug in die Praxis gehalten. Es wird möglich, komplexe physikalische Vorgänge mit rechenintensiven mathematischen Methoden wiederzugeben, womit die Computermodellierung $\mathrm{zu}$ einem gängigen Werkzeug der modernen Ingenieurin und des modernen Ingenieurs wird. Es liegt auf der Hand, dass sich die Rolle von Modellierungssoftware sowie der entwickelnden und anwendenden Personen im Laufe der Zeit maßgeblich geändert hat. Abbott (1991) fasst folgende fünf Generationen zusammen:

Erste Generation - 1950er-Jahre: Bedingt durch die steigende Rechenleistung und die Entwicklung mathematischer Ansätze gewinnt das numerische Lösen algebraischer Gleichungen an Bedeutung. Die Anwendenden sind spezialisierte Expertinnen und Experten, die zugleich in der Entwicklung tätig sind.

Zweite Generation - 1960er-Jahre: Die numerischen Modelle sind für bestimmte Projektanforderungen programmiert. Die Modelle werden von einer Gruppe aus Expertinnen und Experten entwickelt und angewandt.

Dritte Generation - 1970er- \& 1980er-Jahre: Erste Modellsysteme werden von Fachleuten entwickelt und für Anwendende angeboten. Anwendende müssen zumeist keine Entwick- lungsarbeit leisten, jedoch für die Anwendung des numerischen Modells einschlägiges Fachwissen besitzen.

Vierte Generation - 1980er- bis 2000er-Jahre: Softwarepakete werden als Service, inklusive Trainingskurse und Hilfestellungen, oft durch die Entwickelnden kommerziell angeboten. Die Anwendenden werden von Fachleuten des Softwareunternehmens geschult und das erforderliche Wissen für die Anwendung kann gering sein.

Fünfte Generation - seit 2010: Kommerzielle Modellierungssoftware wird dementsprechend weiterentwickelt, um das erforderliche Hintergrundwissen des Anwendenden gering $\mathrm{zu}$ halten. Ein Eingriff in den Modellierungsprozess ist stark eingeschränkt, zumeist wird mit Standardeinstellungen und Parametern der Entwickelnden gearbeitet. Anwendende müssen zumeist nur ein sehr eingeschränktes Wissen über die von der Software verwendeten Methoden haben. In einer weiterführenden Publikation sprechen Abbott und Vojinovic (2009) zusätzlich von einem erkennbaren Trend in Richtung web-basierter Modellierungen (Software-as-a-Service).

Es ist offensichtlich, dass sich die Rollen von Personen in der Entwicklung und Anwendung im Laufe der Zeit stark verändert haben. So waren in den Anfängen der numerischen Modellierung die Anwenderinnen und Anwender auch gleichzeitig die Entwickelnden der Programme und mussten auch das dafür notwendige Fachwissen mitbringen. Heutzutage besteht bei kommerzieller Modellierungssoftware eine klare Trennung zwischen entwickelnden und anwendenden Personen. Dadurch steckt ein Großteil des Fachwissens bei den entwickelnden Personen der Software, welches auf möglichst anwendungsfreundliche Weise, z. B. durch die Auswahl von universellen Standardparametern, numerisch stabilen Lösern etc. bereitgestellt wird. So wird z.B. beim Produkt Discovery Live von ANSYS (ANSYS 2017) damit geworben, die Modellierung für jede Produktentwicklerin und jeden Produktentwickler zugänglich zu machen, auch ohne einschlägiges Grundlagenwissen bei der entwickelnden Person.

\section{Vergleich zwischen frei verfügbarer und kommerzieller Software}

In diesem Kapitel wird der Unterschied zwischen kommerzieller und frei verfügbarer Software diskutiert. Folgende Gegenüberstellungen sind eine Aggregation aus Literatur (Kooths et al. 2003) und eigener Erfahrungen bzw. Meinungen der Autoren.

\subsection{Schaffung von Innovation}

Aus Sicht von Kooths et al. (2003) ist bei kommerzieller Software ein höherer Innovationsgrad gegeben, da aufgrund der Marktsituation bei den entwickelnden Unternehmen große Anreize zu Investition, Weiterentwicklung und Innovation bestehen. Hingegen ist Freeware zumeist weniger lukrativ, was sich in fehlenden Entwicklungsbudgets widerspiegelt. Oft kann Freeware im sich schnell verändernden Markt nicht bestehen und wird nicht weiterentwickelt. Bei Open-Source-Software andererseits steht die Entwicklung allen Beteiligten offen. Das kann ein Vorteil sein, wenn es um eine Vielzahl kleinerer Änderungen geht. Für die Umsetzung größerer Entwicklungskonzepte sind bei kommerzieller Softwareentwicklung jedoch Strukturen gegeben, die das Erlangen einer Marktreife erleichtern.

Nichtsdestotrotz soll darauf hingewiesen werden, dass eine Symbiose zwischen kommerziellen Lösungen und Open-Source-Projekten besteht. Viele kommerzielle Anwendungen sind stark von Open-Source-Komponenten inspiriert oder verwenden Teile davon. Als Beispiel kann die Software Mike Urban von DHI (DHI 2017) genannt werden. Diese Software für die Modellierung urbaner Wasserkreisläufe verwendet Teile der von der United States Environmental Protection Agency (EPA) entwickelten Modelle SWMM (Rossmann 2015) und EPANET (Rossmann 2000). Nicht selten sind bei bekannten OpenSource-Projekten große Firmen tätig oder unterstützen diese finanziell. Als prominentes Beispiel kann der LinuxKernel genannt werden, zu dem Firmen wie Google, Intel, Samsung oder IBM maßgeblich beitragen (Schrape 2015). In seiner Publikation schätzt Schrape (2015) ab, dass mit Stand 2015 ca. 25.000 Personenjahre in die Entwicklung der Linux-Distribution Debian geflossen sind. Vergleicht man auch den Marktanteil von Linux-basierten 


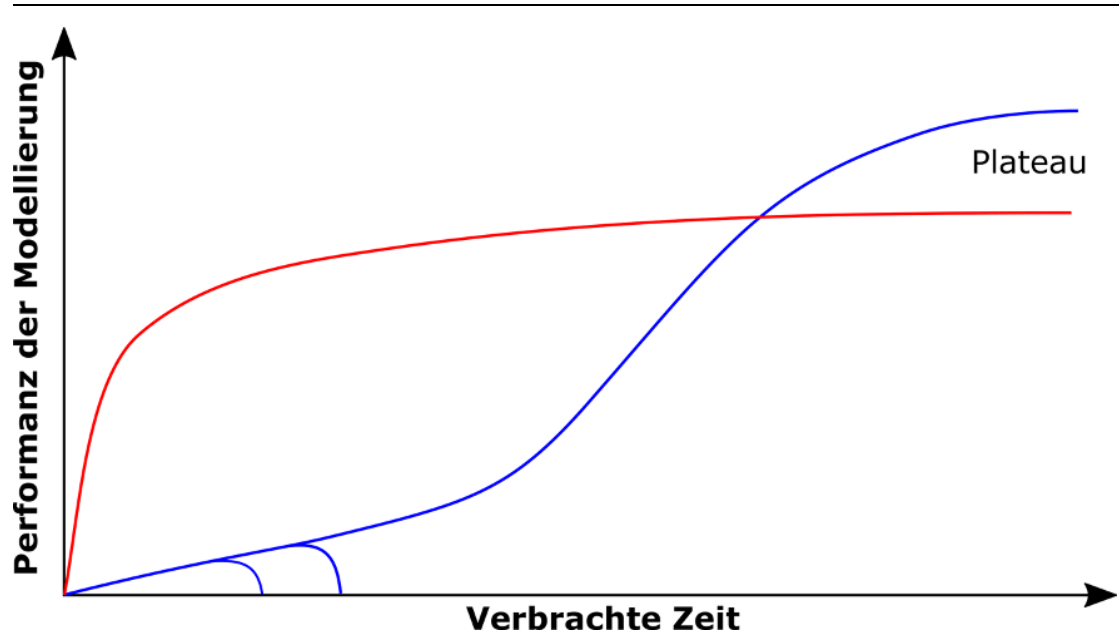

Abb. 1 Verhältnis der verbrachten Zeit zur erzielten Performanz in der Anwendung numerischer Modelle. Rote Linie: kommerzielle Software, blaue Linie: frei verfügbare Software

Betriebssystemen mit kommerziellen Microsoft-Windows-Alternativen am Beispiel von High Performance Computing (HPC) Einrichtungen, zeigt sich, dass Linux einen Marktanteil von $75 \%$ aufweist, wohingegen Microsoft Windows im direkten Vergleich nur 6\% besitzt (IBM 2012).

\subsection{Betrachtungen im Hinblick auf Anwendende}

Generell kann die Aussage getroffen werden, dass die Entwicklung kommerzieller Software publikumsorientiert erfolgt, wohingegen Open-Source-Software und Freeware eher mit Bezug auf die Anforderungen der Entwickelnden erstellt werden. So stehen bei kommerzieller Software beispielsweise Benutzungsfreundlichkeit oder Bedienbarkeit im Vordergrund. Hingegen stehen bei der Entwicklung der frei verfügbaren Alternativen zumeist technische Aspekte an erster Stelle. Dies kann sich in Form von unzureichender Dokumentation, Installationshürden (z. B. Kompilieren der Software) oder fehlendem Support abzeichnen. All das führt $\mathrm{zu}$ einem erschwerten Einstieg in frei verfügbare Modellierungssoftware. Nicht selten geben Einsteigerinnen und Einsteiger, vor allem wenn diese bereits Erfahrungen mit einem kommerziellen Produkt haben, deshalb nach geringer Zeit auf. Abb. 1 zeigt die Lernkurven anhand des Vergleichs der Performanz der Modellierung im Verhältnis zur investierten Zeit. Auf der Abszisse ist die mit der Software verbrachte Zeit aufgetragen, auf der Ordinate ist die Performanz der
Modellierung oder der modellierenden Person dargestellt. Bei kommerzieller Software (rote Linie) zeichnet sich ein schneller Einlernprozess ab. Das Plateau, das als volle Ausnutzung der Kapazitäten des Programms interpretiert werden kann, wird hier relativ schnell erreicht. Im Gegensatz dazu kann am Anfang bei der Benutzung von frei verfügbarer Software (blaue $\mathrm{Li}$ nie) eine flache Lernkurve beobachtet werden. Grund dafür sind zum Beispiel Installationsschwierigkeiten, das oftmals notwendige Einlernen in Dateiformate und Verzeichnisstrukturen sowie das Verstehen der oft vielen numerischen Einstellungsmöglichkeiten. Viele Anwenderinnen und Anwender geben bedingt durch Zeitmangel gerade in dieser Phase auf. Hat man jedoch die Anfangshürden überwunden, kann von einem beschleunigten Lernprozess ausgegangen werden. Das Plateau liegt bei freier Software - speziell bei Open-Source-Produkten - in der Regel höher, da das Modell durch Veränderungen des Quellcodes an projekt- oder forschungsspezifische Anforderungen angepasst werden kann.

\subsection{Standardisierung, Kompatibilität und Updates}

Bei kommerzieller Software streben die entwickelnden Personen eine hohe Standardisierung ihrer Produkte an. Somit kann eine Kompatibilität bei verschiedener Hardware und Software leichter erreicht werden. Als Beispiel kann die Kompatibilität der Software zwischen verschiedenen Betriebssys- temen genannt werden. Des Weiteren ist bei kommerzieller Software häufiger eine Kompatibilität der Programmversionen gegeben, was Anwendenden den Umstieg auf eine neuere Version deutlich erleichtert. Bei frei verfügbarer Software kann es passieren, dass öfter als bei kommerziellen Programmen die Rückkompatibilität der Versionen nicht gegeben ist, was dazu führt, dass nach einem Update bestehende numerische Modelle nicht mehr lauffähig sein können. Bei der softwareübergreifenden Kompatibilität bieten kommerzielle Produkte häufig eine Vielzahl von Dateiformaten an. Jedoch sind Anwendende auf die implementierten Formate beschränkt, was wiederum Einschränkungen bei der Benutzung mit sich bringen kann. Eine speziell bei Open-Source-Software gegebene Gefahr, aber auch möglicher Mehrwert, ist das „Forking“. Forking beschreibt eine Abspaltung von zwei oder mehreren Folgeprojekten vom Mutterprojekt. Die Entwicklungen werden unabhängig getätigt und weiterverbreitet, was $\mathrm{zu}$ einer Streuung des Wissens und weiterführend auch der Kapazitäten führt. Ein berühmtes Beispiel dafür ist die Open-Source-CFD-Software OpenFOAM (Weller et al. 1998).

\subsection{Veränderbarkeit, Einblick in den Code und Kopplung}

Aufgrund des Eigentumsrechts bei kommerzieller Software und Freeware haben Anwendende keinen Einblick in den Quellcode und daher ist keine Veränderbarkeit gegeben. Hingegen sind bei Open-Source-Software eine beliebige Anpassung des Quellcodes und eine Weiterverbreitung möglich. Damit sind Open-Source-Produkte gerade an Universitäten attraktiv, da auf deren Basis Forschungstätigkeiten durchführbar sind.

\section{5 Überlegungen zu den Gesamtkosten}

Neben den klar ersichtlichen Lizenzkosten fließen im Zuge der finanziellen Entscheidung zwischen frei verfügbarer und kommerzieller Software noch weitere Faktoren ein. Diese sind zum Beispiel die aufgewandte Zeit für die Modellerstellung sowie die Einlernphase der Anwenderin und des Anwenders, Fehlerbehebungen sowie Qualitätssicherung. Somit ist gerade in privatwirt- 

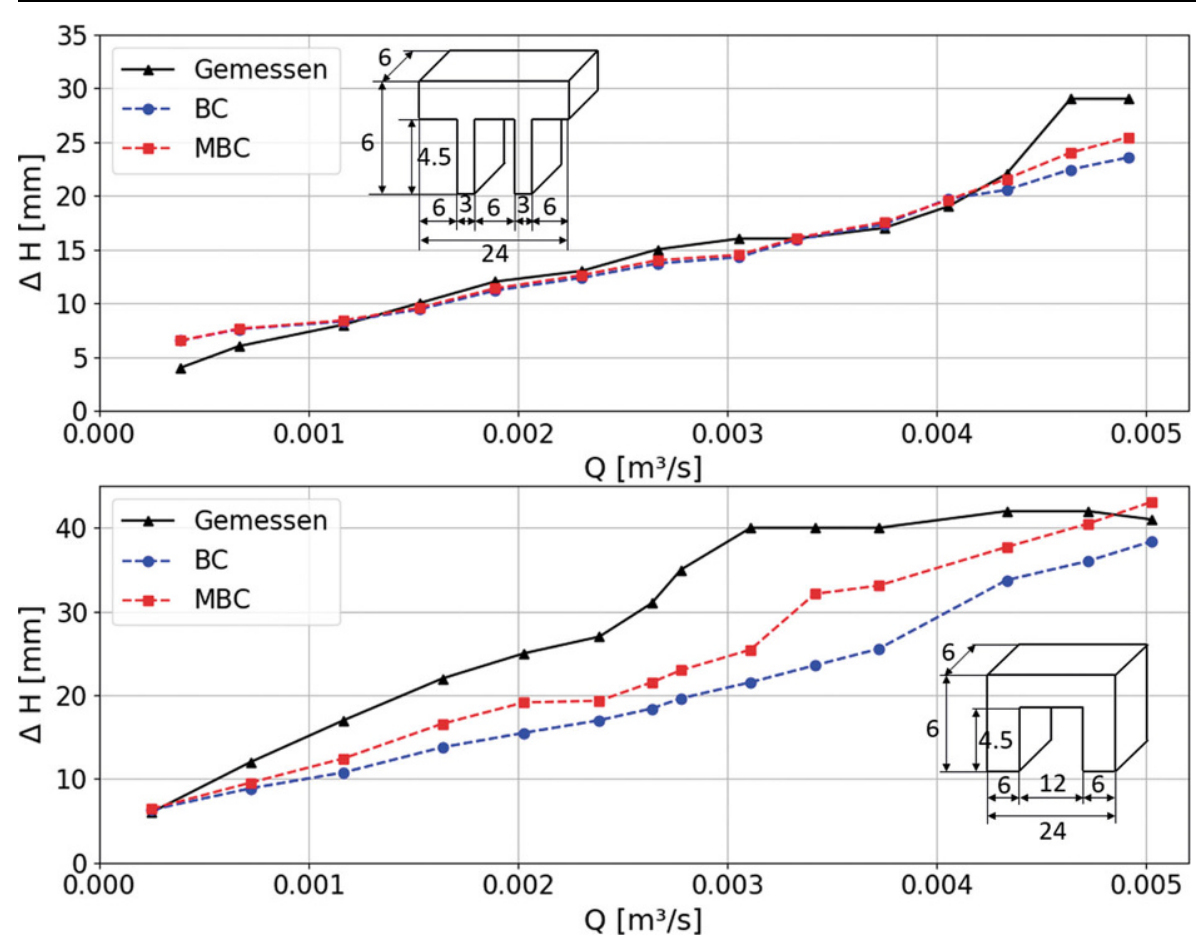

Abb. 2 Validierung desBrückenmoduls, Vergleichvonsimuliertenund gemessenenWasserspiegeldifferenzen; EinheitderBrückenschemas in $\mathrm{cm}$

schaftlichen Unternehmen bei Überlegungen zum Einsatz von Open-SourceProgrammen abzuwägen, ob die dafür notwendigen Ressourcen gegeben sind, um praxisbezogene Aufgabenstellungen effizient bearbeiten zu können.

\section{Beispiele für Open-Source- Anwendungen}

\subsection{Berücksichtigung von}

hydraulischen Verlusten am Beispiel der Implementierung eines Brückenmoduls

Hydraulische Verluste können in Form von Fließwiderständen in den 2D SaintVenant-Gleichungen berücksichtigt werden. Durch die Modifizierbarkeit des Quellcodes bieten deshalb OpenSource-Modelle eine attraktive Möglichkeit, um anwendungsspezifische hydraulische Verluste, z. B. Strömungsturbinen (Joly et al. 2015), abzubilden. In diesem Kapitel wird die Implementierung von hydraulischen Verlusten in das Open-Source-Modell Telemac-2D (Hervouet 2007) für die Berücksichtigung von Brückenbauwerken beschrieben. Die Ansätze von Ratia et al. (2014) dienen dabei als Grundlage.

In 2D tiefengemittelten Modellen wird der Einfluss von Brücken zumeist mittels einer der drei folgenden Methoden berücksichtigt:

- Brücken als innere Randbedingungen: z.B. geometrische Abbildung der Fahrbahndecke in Kombination mit Durchlässen. Diese Methode wird derzeit in Telemac-2D verwendet.

- Geometrische Abbildung: Abbildung der Brückenpfeiler und Widerlager in Kombination mit Verlustansätzen bei eingestauter Fahrbahndecke.

- Berücksichtigung des gesamten Brückenbauwerks mittels hydraulischer Verluste.

Das in Telemac-2D neu implementierte Brückenmodell berücksichtigt hydraulische Verluste als Funktion von Abflussgrößen und Parametrisierungen der Brückengeometrie. Der lokale Fließwiderstand wird basierend auf den BordaCarnot'schen Verengungs- und Aufweitungsverlusten berechnet und direkt in der Impulsgleichung angesetzt. In Ratia et al. (2014) wurde des Weiteren ein aus Laborversuchen ermittelter Modifikationsbeiwert ermittelt, welcher die originale Borda-Carnot-Formulierung verbessern soll. Dieser Beiwert ist abhängig von den flussauf und flussab berechneten Froude-Zahlen, dem Verhältnis der von der Brücke belegten Fläche im Profilquerschnitt, der unbe- einflussten Querschnittsfläche (in Abhängigkeit von der Wassertiefe) sowie einem empirisch ermittelten Beiwert für nicht, teilweise und vollständig eingestaute Brücken.

Die Validierung des implementierten Ansatzes erfolgt anhand von in Ratia et al. (2014) durchgeführten Modellversuchen. Dabei wurden 22 Versuche in einem $24 \mathrm{~cm}$ breiten Glasgerinne mit verschiedenen Brückengeometrien und Randbedingungen durchgeführt, für welche die Differenzen zwischen Unter- und Oberwasserspiegel ausgewertet wurden. Abb. 2 vergleicht für zwei ausgewählte Brückengeometrien die gemessenen (schwarze Linie) mit den numerisch simulierten Wasserspiegeldifferenzen (MBC, rote Linie) bei variierendem Durchfluss. Verglichen wird zudem der klassische Borda-Carnot'sche (BC, blaue Linie) Ansatz. Die Ergebnisse zeigen, dass bei der Geometrievariante 1 (Abb. 2 oben) eine gute Übereinstimmung über das gesamte Abflussspektrum gegeben ist. Hingegen ist bei der Geometrievariante 2 (Abb. 2 unten) eine Unterschätzung der Wasserspiegeldifferenzen für die mittleren Abflüsse zu erkennen.

Generell konnte mit dem implementierten Verlustansatz zur Abbildung komplexer Brückengeometrien eine gute Übereinstimmung zwischen 


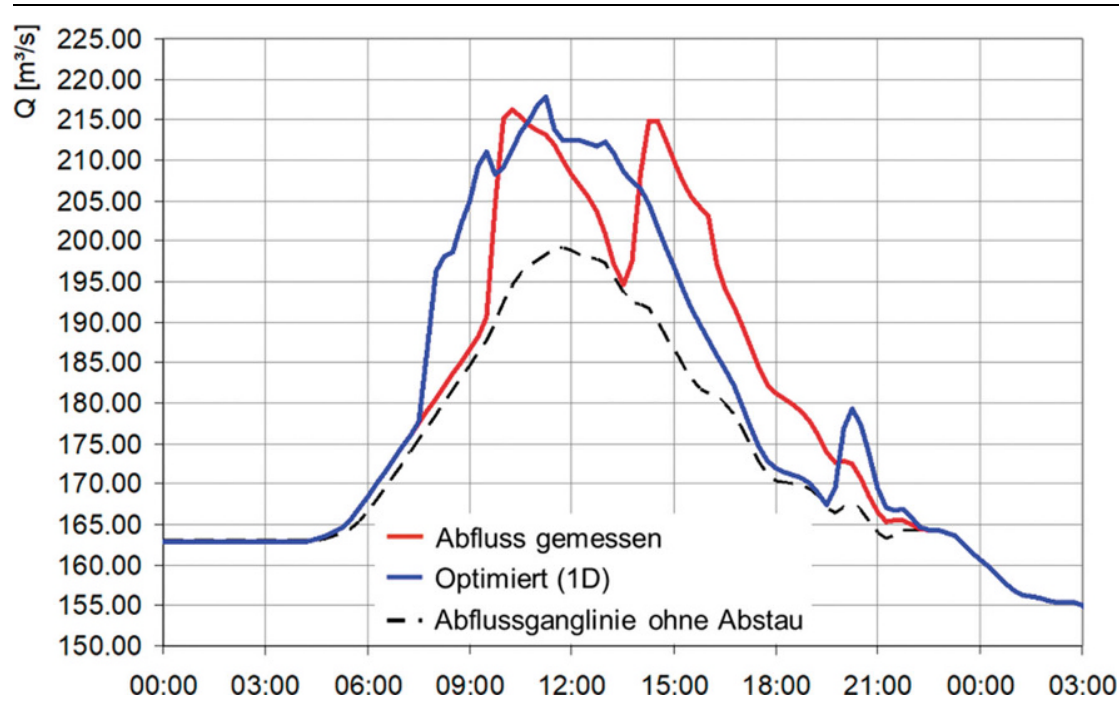

Abb. 3 1D numerische Optimierung der Staukettenregelung von zwei Wasserkraftwerken bei Hochwasserereignissen (Dorfmann 2006)

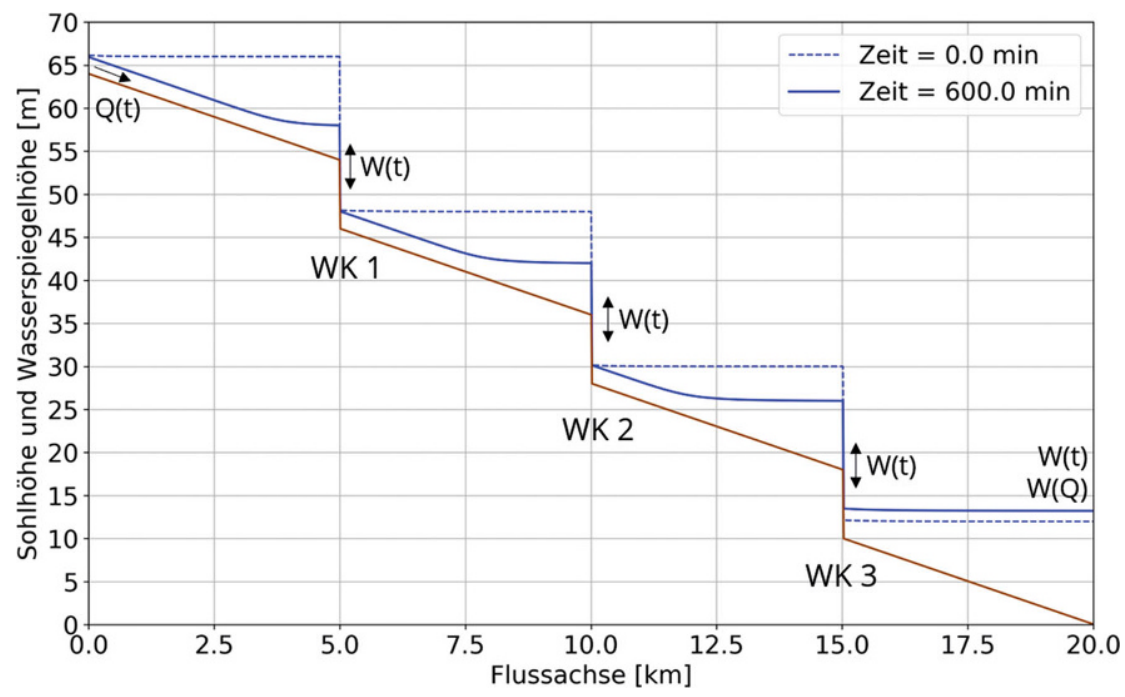

Abb. 4 Simulation einer Staukette mit Telemac-2D unter Verwendung interner hydraulischer Randbedingungen

den Messungen und der Numerik erzielt werden. Des Weiteren wurden im Vergleich zum klassischen Telemac-2DAnsatz weniger Modellinstabilitäten beobachtet.

\subsection{Implementierung von internen Randbedingungen in ein tiefengemitteltes 2D Modell}

Viele Flüsse in Mitteleuropa sind in deren Längskontinua durch Wasserkraftwerke bzw. Stauketten unterbrochen. Die Wasserspiegel und Abflüsse an Kraftwerken werden durch Betriebseinrichtungen geregelt, sei es im Normalbetrieb als auch bei Hochwasserer- ses, erlauben. Ein Beispiel für eine 1D numerische Staukettenoptimierung in einer Flussstrecke mit zwei Wasserkraftwerken ist in Abb. 3 dargestellt.

In 1D-Modellen von Stauräumen mit komplexer Geometrie werden allerdings die durch die Wehrsteuerung hervorgerufenen zeitlichen Volumenänderungen oftmals mit Ungenauigkeiten simuliert, da z.B. eventuell auftretende großräumige Rückströmungen nicht berücksichtigt oder Volumina, z.B. in seitlichen Buchten, zeitlich falsch aktiviert werden. Heutzutage können mit tiefengemittelten 2D-Modellen Flussstrecken mit Längen über $50 \mathrm{~km}$ simuliert werden. 2D-Simulationen erlauben im Vergleich zum 1DAnsatz eine weitaus exaktere Abbildung des instationären Strömungsverhaltens in komplexen Geometrien, jedoch haben die meisten kommerziellen 2DSoftwarepakete keine internen Randbedingungen implementiert oder ein Zusatzmodul muss teuer dazugekauft werden. Im Folgenden wird ein einfacher, aber akkurater Ansatz nach Dorfmann (2019) für die Implementierung interner hydraulischer Randbedingungen in das Open-Source-Modell Telemac-2D vorgestellt. Dabei werden geregelte Bauwerke als interne Randbedingungen abgebildet. Das Berechnungsgebiet wird in Teilnetze aufgeteilt, welche an den offenen Rändern über zeitlich veränderliche Wasserspiegel bzw. Wasserstands-Abfluss-Beziehungen gekoppelt werden können. Der in Telemac-2D implementierte Ansatz wurde anhand mehrerer Datensätze mittels eines numerischen 1D-Modells validiert. Abb. 4 zeigt exemplarisch die 2D-Berechnung einer Hochwasserwelle in einer $20 \mathrm{~km}$ langen Flussstrecke mit drei Wasserkraftwerken (WK). Während im Normalbetrieb die Wasserkraftwerke das Stauziel halten, senken diese bei einem Hochwasserereignis die Stauräume nach jeweiligen Vorschriften ab.

Die Implementierung interner hydraulischer Randbedingungen in ein numerisches 2D-Modell erlaubt die gesamte Betrachtung einer Flussstrecke mit geregelten Bauwerken. Als Beispiele für Anwendungen seien genannt: gesamtheitliche Abbildung der instationären Strömungsvorgänge und Überflutungen bei einem Hochwasserereignis, Simulation von Schwall- und Sunkwellen durch graduelles Schließen oder Öffnen von Verschlussorganen, Simulation von Wendepegelsteuerungen, Optimierung von Kraftwerkssteuerun- 
gen bzw. Wehrregelungen hinsichtlich Stauraumspülungen und Sedimentdurchgängigkeit, Einsatz in gekoppelten hydrologisch-hydraulischen Modellen.

\subsection{Beispiel serielle Modellkopplung}

Ein erkennbarer Trend, der schon von Knight (2013) angesprochen wurde, ist die umfassende Betrachtung von Prozessen in Einzugsgebieten. So sind physikalische, biologische und sozioökonomische Prozesse eng miteinander verbunden, interagieren miteinander und müssen deshalb in ihrer Gesamtheit betrachtet werden. Diese Notwendigkeit zeigt sich in der Erarbeitung von Standards sowie der Entwicklung von Workflow-Paketen und integrierten Modellierungsumgebungen. Diese wiederum bilden die Grundlage für aktuelle Forschungsthemen, wie z.B. Unsicherheitsanalysen, Datenassimilierung, Echtzeit-Steuerungen oder operationelle Vorhersagemodelle. Bastin et al. (2013) unterscheiden drei Arten der integrierten Modellierung:

(i) Standardsprachen und -schnittstellen: Die Kopplung von Modellkomponenten erfolgt hier über einheitliche Programmiersprachen, z. B. über Python-APIs oder Formatstandards wie OpenMI (Gregersen et al. 2007).

(ii) Workflow-Pakete: Diese koppeln einzelne Programmkomponenten in serieller Weise. Als Beispiel für ein OpenSource-Workflow-Paket kann ecFlow (Varela und Bonet 2017) genannt werden.

(iii) Frameworks: Diese sind Modellierungsumgebungen, die einfache Kopplungen innerhalb eines bestimmten Softwarepakets erlauben. Zumeist werden das Framework und das Softwarepaket von demselben Unternehmen entwickelt. Als Beispiel kann das OpenSource-Framework Delta Shell von Deltares (Donchyts und Jagers 2010) genannt werden.

Im Rahmen des EFFORS-Projekts (Gegenleithner et al. 2018) wurde auf Basis der Open-Source-Workflow-Software ecFlow ein Steuerprogramm für die operationelle Hochwasservorhersage entwickelt. ecFlow wird vom European Centre for Medium-Range Weather Forecasts (ECMWF) entwickelt und für die operationelle Wettervorhersage benutzt. Die ecFlow-Umgebung bietet für operationelle Systeme Funktionalitäten an, wie z.B. automatisierte Neustartmöglichkeiten, Statusberichte der

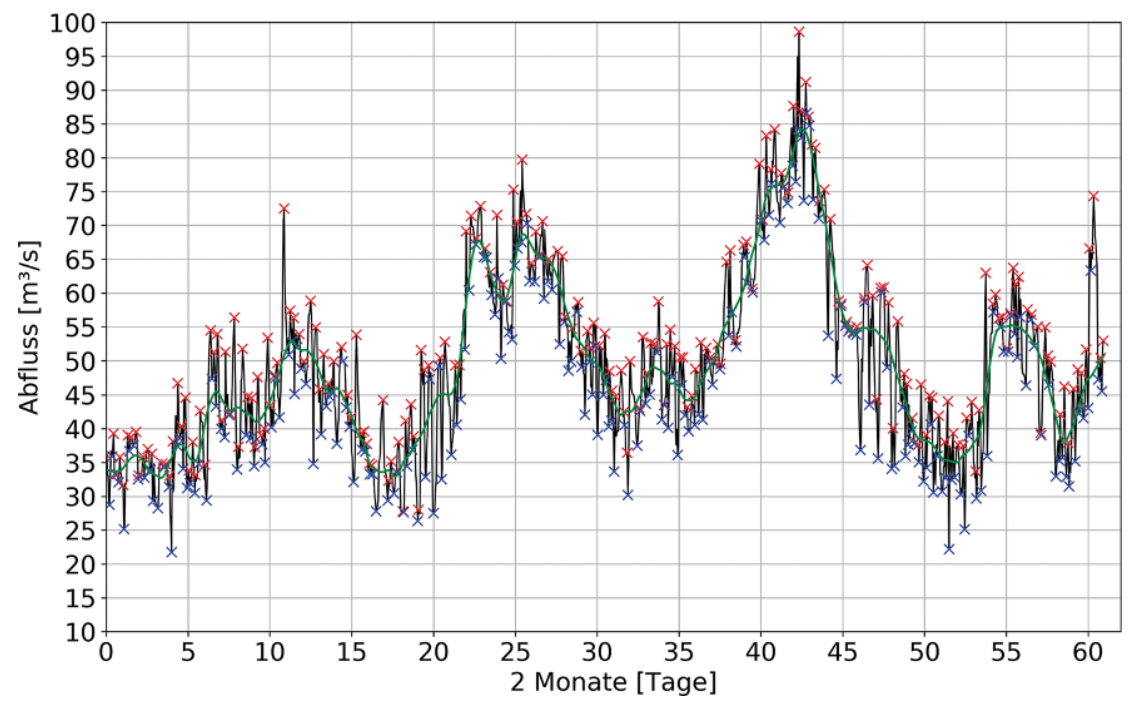

Abb. 5 Analyse einer gemessenen, durch Schwall-Sunk beeinflussten Abflussganglinie, Schwallspitzen rot und Sunkminima blau gekennzeichnet

einzelnen Prozesse, Prävention von im operationellen Betrieb entstehenden Coderelikten durch Vergabe von Passwörtern an die Module etc. Auf Basis der Grundfunktionalitäten wird über eine Python-API eine serielle Kopplung der Module realisiert. Die dynamische Umsetzung des Steuerprogramms erlaubt eine einfache Anpassung von Prognosehorizonten, Modellupdates, Softwareupdates etc. über im JSONFormat vorliegende Steuerdateien. Im Folgenden wird der Ablauf der Programmkopplung beschrieben:

Die Prognose im EFFORS-System wird täglich um 00:00 gestartet. Die Steuerdateien werden eingelesen und die Prognoseintervalle dynamisch initialisiert. Das bedeutet, dass zum Beispiel bei einem Updateintervall der Prognose von $1 \mathrm{~h} 24$ Teilprognosen erstellt werden (00:00, 01:00 etc.). Täglich um 00:00 werden auch automatisiert Updates von Programmen und Modellen durchgeführt, um eine unterbrechungsfreie Prognose zu gewährleisten. Anschließend wird die Prognose gestartet. Dazu werden zuerst die notwendigen Eingangsdaten, z. B. meteorologische Daten, Echtzeitmessungen von Pegelmessstellen etc. gesammelt. Die Eingangsdaten werden prozessiert, sodass diese vom hydrologischen Modell verarbeitet werden können. Als nächster Schritt erfolgt die numerische Prognose (Hydrologie, 1D- und 2D-Hydrodynamik). In der Datenaufbereitung werden die Ergebnisse schließlich ausgewertet, dargestellt, Warnungen generiert und archiviert.

\subsection{Beispiel für die Auswertung von Messdaten}

Im Wasserbau, wie in anderen Disziplinen, werden die Herausforderungen bezüglich der $\mathrm{zu}$ bearbeitenden Messdatenmengen aufgrund der sich kontinuierlich entwickelnden und ausgereifteren Messmethoden immer größer. Genannt seien hier beispielhaft die Bearbeitung bzw. Analyse von LidarDaten, Satellitendaten z.B. für Landnutzungsklassifizierung, langjährigen Zeitreihen oder hochaufgelösten dreidimensionalen Geschwindigkeitsmessungen. Im Folgenden wird die Analyse einer Durchflussmessreihe mit Schwall-Sunk-Abfolgen diskutiert. Auf den ersten Blick mag die Durchführung einer statistischen Analyse der Abflüsse, Abflussgradienten, Frequenzen der Schwall-Sunk-Abfolgen oder Dauer der jeweiligen Schwall-SunkPerioden einfach erscheinen. Aber welche Software bzw. welche Methoden sind dafür wirklich geeignet? Fündig kann man in Open-Source-PythonBibliotheken werden. So erlaubt die Anwendung von Algorithmen aus der elektrotechnischen Signalverarbeitung in Kombination mit nicht-parametrischen Regressionen sowie Filtermethoden semi-automatisierte, effiziente und akkurate Schwall-Sunk-Analysen langer Zeitreihen. Abb. 5 zeigt eine gemessene Abflussmessreihe von einer SchwallSunk-beeinflussten Flusstrecke und die Analyse der detektierten Schwallspitzen (rot) sowie Sunkminima (blau), auf 
Basis derer weitere abgeleitete Größen bestimmt werden können.

\section{Diskussion}

Die generelle Frage, welcher Softwaretyp (frei verfügbar oder kommerziell) verwendet werden soll, ist nicht einfach zu beantworten und hängt stark von der Anwendung und den Anwendenden ab. Für viele Anwenderinnen und Anwender von frei verfügbarer Software ist das schon eine Prinzipfrage, da diese von kommerziellen Interessen der Unternehmen unabhängig sein wollen und den Gedanken zu einem gemeinsamen Projekt etwas beizutragen höchst ansprechend finden - „Open Source Spirit“. Lässt man persönliche Präferenzen außen vor, können die Stärken der jeweiligen Typen folgendermaßen zusammengefasst werden:

Kommerzielle Softwareprodukte bieten durch ihre publikumsorientierte Entwicklung viele Vorteile hinsichtlich Benutzungsfreundlichkeit, Support etc. Zumeist sind diese auch kompatibler, was ein Update der verwendeten Version erleichtert. Das marktwirtschaftliche Interesse treibt auch nicht selten Entwicklungsinnovationen an. Generell ist bei großen kommerziellen Softwareunternehmen ein größeres Entwicklungsbudget vorhanden.

Die Stärken von Open-Source-Software liegen in der hohen Flexibilität bedingt durch die Veränderbarkeit hinsichtlich vorhandener Anforderungen. Des Weiteren bieten viele Open-SourceEntwickler standardisierte Schnittstellen, was eine softwareübergreifende ganzheitliche Modellierung von Prozessen erlaubt, z. B. Kopplung physikalisch basierter und sozioökonomischer Modelle.

Die numerische Modellierung hydrodynamischer Prozesse ist heutzutage eine der Hauptaufgaben sowohl in der wasserbaulichen Praxis als auch in der Forschung. Solche Modellierungen erlauben Einsicht in komplexe physikalische Prozesse, von der Einzugsgebietsskala - ganzheitliche Betrachtung von Meteorologie, Hydrologie und Hydrodynamik und die daraus abgeleiteten sozioökonomischen Auswirkungen - bis hin zu kleinskaligen Untersuchungen der Turbulenz. Nichtsdestotrotz sind die schnelle Entwicklung und Verfügbarkeit numerischer Modellierung auch kritisch zu sehen. Vor allem bei kommerzieller Software ist der Trend zu erkennen, dass die Programme immer anwendungsfreundlicher werden und diese somit weniger Einsicht und Eingriff erlauben - z.B. Voreinstellungen wichtiger numerischer Parameter.

In einem Grundsatzpapier schreibt die International Association for HydroEnvironment Engineering and Research (IAHR), dass, bedingt durch die zunehmende Einführung neuer Hilfsmittel wie der numerischen Modellierung, die Streuung des Wissens in Spezialisierungen mündet, was im Rückschluss einen Verlust des gesamtheitlichen Überblicks über hydraulische Problemstellungen bedeutet (IAHR 1993; Knight 2013). Wie Knight (2013) des Weiteren folgert, birgt dies die Gefahr, dass numerischen Modellierungen blind vertraut wird und angenommen wird, dass die Visualisierung von Modellierungsergebnissen eine wahre Repräsentation der physikalischen Prozesse darstellt. Anwendende sollten sich jedenfalls der Fähigkeiten, Unsicherheiten und Grenzen der verwendeten Software und grundsätzlich der numerischen Modellierung bewusst sein.

\section{Zusammenfassung}

Das Lösen wasserbaulicher Problemstellungen mithilfe numerischer Modelle hat in den letzten Jahrzehnten signifikant an Bedeutung gewonnen. So ist sowohl bei kommerziellen Softwareprodukten als auch bei frei verfügbarer Software eine stetige innovative Entwicklung hinsichtlich numerischer Methoden, aber auch Bedienungsfreundlichkeit zu beobachten. Nach wie vor wird in der Praxis besonders für 2D und 3D numerische Aufgabenstellungen vornehmlich Software kommerzieller Softwareentwicklungsunternehmen eingesetzt, da diese meist eine schnellere Lernkurve aufweisen und umfangreicher Support angeboten wird. Frei verfügbare, speziell Open-Source-Modellierungssoftware, fristet in der Praxis generell ein Nischendasein und wird vor allem in öffentlichen Institutionen für Forschungstätigkeiten eingesetzt. Vorteile und Nachteile sind natürlich auf beiden Seiten zu finden, wobei bei kommerzieller Software besonders die Bedienendenfreundlichkeit, hingegen bei Open-Source-Software die Anpassung an projektspezifische Anforderungen hervorgehoben werden kann.

Auf der einen Seite gewinnen, aufgrund der Verfügbarkeit von Rechenleistung oder Cloud-Computing, komplexe 3D-Modellierungen für die Un- tersuchung kleinskaliger physikalischer Prozesse an Bedeutung und werden neben der Forschung auch vermehrt in der Praxis angewandt. Auf der anderen Seite erfordert die zunehmend gesamtheitliche, einzugsgebietsbezogene Betrachtung physikalischer, biologischer und sozioökonomischer Prozesse die Wechselwirkung verschiedener Modelle, welche aus grundsätzlich völlig verschiedenen Fachbereichen stammen können. Die Entwicklung von Kopplungsmethoden, seien es standardisierte Schnittstellen, Workflows oder integrierte Modellsysteme, wurde und wird vor allem von Open-Source-Initiativen und auch großen kommerziellen Softwareunternehmen vorangetrieben. Solche Systeme bilden die Basis für Echtzeit-Steuerungen oder operationelle Vorhersagemodelle.

Ein weiterer Trend ist in Richtung nicht-physikalisch basierter Modellierungs- und Analysemethoden für wasserbauliche Fragestellungen zu erkennen. Aktuelle Forschungsthemen wie methodische Unsicherheitsanalysen, Datenassimilierungsmethoden oder Machine Learning werden in naher Zukunft Einzug in die Praxis halten. Aus Sicht der Autoren bringen diese Entwicklungen neue Herausforderungen für den Wasserbau mit sich und fordern von Anwendenden die kontinuierliche Aneignung von Basiswissen, um Ergebnisse, sei es aus kommerziellen als auch aus frei verfügbaren Programmen, analysieren und richtig interpretieren zu können.

Funding Open access funding provided by Graz University of Technology.

Open Access Dieser Artikel wird unter der Creative Commons Namensnennung 4.0 International Lizenz veröffentlicht, welche die Nutzung, Vervielfältigung, Bearbeitung, Verbreitung und Wiedergabe in jeglichem Medium und Format erlaubt, sofern Sie den/die ursprünglichen Autor(en) und die Quelle ordnungsgemäß nennen, einen Link zur Creative Commons Lizenz beifügen und angeben, ob Änderungen vorgenommen wurden.

Die in diesem Artikel enthaltenen Bilder und sonstiges Drittmaterial unterliegen ebenfalls der genannten Creative Commons Lizenz, sofern sich aus der Abbildungslegende nichts anderes ergibt. Sofern das betreffende Material nicht unter der genannten Creative 
Commons Lizenz steht und die betreffende Handlung nicht nach gesetzlichen Vorschriften erlaubt ist, ist für die oben aufgeführten Weiterverwendungen des
Materials die Einwilligung des jeweiligen Rechteinhabers einzuholen.
Weitere Details zur Lizenz entnehmen Sie bitte der Lizenzinformation auf http://creativecommons.org/licenses/ by/4.0/deed.de.

\section{Literatur}

Abbott, M. B. (1991): Hydroinformatics: Information Technology and the Aquatic Environment. Avebury Technical, Aldershot, UK.

Abbott, M. B., Vojinovic, Z. (2009): Applications of numerical modelling in hydroinformatics. Journal of Hydroinformatics, 11(3-4), 308-319.

Ansys (2017): Welcome to ANSYS Discovery Live [Video file]. Available via https://www.youtube. $\mathrm{com} /$ watch? $\mathrm{v}=\mathrm{Hl}$ jySucPHWk, Accessed April 20, 2020

Bastin, L., Cornford, D., Jones, R., Heuvelink, G. B., Pebesma, E., Stasch, C., ... Williams, M. (2013): Managing uncertainty in integrated environmental modelling: The UncertWeb framework. Environmental Modelling \& Software, 39 116-134

Copeland, B. J. (2006): The Modern History of Computing. Available via https://plato.stanford. edu/entries/computing-history/\#Col, Accessed April 20, 2020

DHI (2017): MIKE Urban User Guide. Danish Hydraulic Institute, Horsolm

DiBona, C., Ockman, S. (1999): Open sources: Voices from the open source revolution. O'Reilly Media, Sebastopol, US

Donchyts, G., Jagers, B. (2010): DeltaShell—an open modelling environment. International Congress on Environmental Modelling and Software, Ottawa, Canada

Dorfmann, C. (2006): Optimierung der Ab- und Aufstauvorgänge bei Stauraumspülungen an der Oberen Mur, Institut für Wasserbau und Wasserwirtschaft, Technische Universität Graz

Dorfmann, C. (2019): Internal boundary conditions for Telemac. 26th Telemac-Mascaret User
Conference, Centre Européen de Recherche et de Formation Avancée en Calcul Scientifique (Cerfacs), Toulouse, France.

Gegenleithner, S., Dorfmann, C., Zenz, G. Band, J., Wölfelmaier, F. (2018): Development of the EFFORS service for operational flood forecasting. 25th Telemac-Mascaret User Conference, Centre for Environment, Fisheries and Aquaculture Science (Cefas), Norwich, UK Gregersen, J. B., Gijsbers, P. J. A., Westen, S. J. P. (2007): OpenMI: Open modelling interface. Journal of hydroinformatics, 9(3), 175-191

Hervouet, J. M. (2007): Free surface flows: Modelling with the finite element methods. Wiley IBM Systems and Technology Group (2012) The hidden costs of open source-Rethinking the economics of infrastructure software, $\mathrm{Ar}$ monk, New York, US

Joly, A, Pham, C. T., Andreewsky, M., Saviot, S. Fillot, L. (2015): Using the DRAGFO subroutine to model Tidal Energy Converters in Telemac2D. Telemac User Club 2015, Oct 2015, Warrington, United Kingdom. hal-01702806 Knight, D. W. (2013): River hydraulics-a view from midstream. Journal of Hydraulic Research, 51(1), 2-18

Kooths, S., Langenfurth, M., Kalwey, N. (2003): Open Source-Software eine volkswirtschaftliche Bewertung, University of Münster

Moore, G. E. (1965): Cramming more components onto integrated circuits. Electronic, 38(8), 114-117

IAHR (1993): IAHR looking into sustainable development: Research agenda and future topics of concern. A Muller, ed. IAHR, Delft
Open Source Initiative (2007): The Open Source Definition. Available at https://opensource.org/ docs/osd. Accessed April 20, 2020

Ratia, H., Murillo, J., García-Navarro, P. (2014): Numerical modelling of bridges in 2D shallow water flow simulations. International Journal for Numerical methods in fluids, 75(4), 250-272.

Rossman, L. A. (2000): EPANET 2 User's Manual. US EPA National Risk Management Research Laboratory. Cincinnati, Ohio, USA

Rossman, L. A. (2015): Storm Water Management Model User's Manual Version 5.1. US EPA National Risk Management Research Laboratory. Cincinnati, Ohio, USA

Schrape, J.-F. (2015): Open Source Softwareprojekte zwischen Passion und Kalkül (No. 2015-02). Stuttgarter Beiträge zur Organisations- und Innovationsforschung, SOI Discussion Paper Varela, D., Bonet, A. (2017): ecFlow. European Centre for Medium-Range Weather Forecasts

Weller, H. G., Tabor, G., Jasak, H., Fureby, C (1998): A tensorial approach to computational continuum mechanics using object-oriented techniques, Computers in Physics, 12(6), $620-631$

Hinweis des Verlags Der Verlag bleibt in Hinblick auf geografische Zuordnungen und Gebietsbezeichnungen in veröffentlichten Karten und Institutsadressen neutral. 